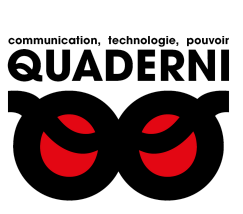

\title{
Quaderni
}

Communication, technologies, pouvoir

95 | Hiver 2017-2018

Logiques numériques des radicalisations

\section{Les radicalisations. Culture et postmodernité}

Avant-propos

Virginie Tournay, Emmanuel Taïeb et Julien Giry

\section{(2) OpenEdition}

Journals

Édition électronique

URL : https://journals.openedition.org/quaderni/1134

DOI : 10.4000/quaderni.1134

ISSN : 2105-2956

Éditeur

Les éditions de la Maison des sciences de l'Homme

Édition imprimée

Date de publication : 5 février 2018

Pagination : 5-12

Référence électronique

Virginie Tournay, Emmanuel Taïeb et Julien Giry, «Les radicalisations. Culture et postmodernité », Quaderni [En ligne], 95 | Hiver 2017-2018, mis en ligne le 05 février 2018, consulté le 03 septembre 2021. URL : http://journals.openedition.org/quaderni/1134; DOI : https://doi.org/10.4000/quaderni. 1134 


\section{$D$ ossier}

\section{Les radicalisations.}

\section{Culture et}

postmodernité

Virginie

Tournay

CNRS / Sciences Po

CEVIPOF

\section{Emmanuel \\ Taïeb}

Institut Universitaire de France Sciences Po Lyon / Triangle

\section{Julien}

Giry

Université Rennes 1

IDPSP
Un État est d'autant plus fort qu'il peut conserver en lui ce qui vit et agit contre lui.

Paul Valéry, Mauvaises pensées et autres.

Démarrer l'avant propos d'un numéro des Quaderni consacré à la place des technologies numériques dans les radicalisations par une citation de Paul Valéry sur la force de l'État peut sembler décalé si on inscrit ce type d'engagement sous le regard exclusif de la sociologie de l'action collective et des formes de socialisation relationnelle. La tonalité générale de ce dossier consiste à conjuguer les acquis de ces approches en saisissant sous un angle culturel la radicalisation entendue comme un processus [Steiner et Önnerfors, 2018] cognitif et politique ${ }^{1}$. En d'autres termes, il s'agit d'appréhender la notion de radicalisation comme un univers de sens, fixateur de normes et de valeurs, ou plutôt de contre-valeurs, de contrerécits, face au récit démocratique et plus singulièrement, de la citoyenneté républicaine. Ce constat suppose dès lors d'intégrer les organisations et la matérialité - les nouveaux médias numériques notamment - mobilisées dans les entreprises de radicalisation, comme des composants essentiels de cette culture. La transmission de cette culture engage un certain type de défiance vis-à-vis du socle républicain et des États-nations.

Cette posture analytique peut paraître contreintuitive car les processus de radicalisation sont des événements de rareté statistique, et par définition ils ne constituent pas la norme, comme le souligne à juste titre Gérald Bronner dans ce dossier. Aussi, il ne saurait être question d'assimiler les manifestations contemporaines de la radicalisation à des mouvements culturels contestataires ou contre-culturels [Bell, 1976] 
tant pour des raisons liées au contenu qu'au processus d'engagement. On est plutôt en présence de logiques transactionnelles proches des dérives sectaires ${ }^{2}$ qui peuvent conduire au bout du compte à l'usage de la violence [Crettiez, 2016]. Il est également intéressant de considérer la radicalisation, qu'elle soit idéologique, politique et/ou religieuse [Benraad 2018a] comme l'expression d'une "insécurité culturelle». L'insécurité concerne la définition de ce qu'est la démocratie, la société française aujourd'hui face aux bouleversements mondiaux [Bouvet, 2015]. On peut la rapprocher d'une « guerre culturelle » [Thomson, 2010], notamment pour ceux qui refusent la sécularisation et l'autonomie des sociétés modernes.

Plutôt que de réduire la radicalisation violente à des (mono)causalités psychologiques, il est nécessaire de décrypter l'univers de sens contenu dans les messages radicaux, ou qui invitent à la radicalisation, et dans leur réception. Par exemple, la propagande djihadiste correspond à un modèle de production continue du sacré qui abolit toute forme de régulation non-violente avec le monde profane. Elle renvoie à la filiation permanente avec un âge d'or mythique et fantasmé ainsi qu'à la désignation mortifère de multiples ennemis. Elle revendique le désir d'un ordre nouveau, une contre-modernité que les modernisateurs du monde profane auraient trahi [Chassignolles, 2016, p. 146-147]. L'analyse socio-culturelle des contenus devient alors essentielle pour déterminer éventuellement les outils institutionnels de lutte contre la radicalisation et imaginer de nouvelles stratégies.

\section{Médias numériques et évolution sémantique du mot « radicalisation »}

La sémantique du mot « radicalisation » a évolué significativement ces quarante dernières années. Elle est aujourd'hui alimentée par une représentation individualisante des phénomènes de radicalisation [Guibet-Lafaye et Rapin, 2017]. Appréhendée dans sa dimension processuelle, la définition de la radicalisation avancée par Xavier Crettiez (p. 712) rend simultanément compte des réseaux d'acteurs, de la matérialité et des éléments symboliques véhiculés par ce terme. On l'entend comme "l'adoption progressive et évolutive d'une pensée rigide, vérité absolue et non négociable, dont la logique structure la vision du monde des acteurs, qui usent pour la faire entendre des répertoires d'action violents, le plus souvent au sein de structures clandestines, formalisées ou virtuelles, qui les isolent des référents sociaux ordinaires et leur renvoient une projection grandiose d'eux-mêmes ».

Si les phénomènes d'emprise, d'isolement et de «manipulation mentale » observés dans la radicalisation sont à rapprocher des dérives sectaires, sa définition a évolué dans la presse. Elle n'est plus décrite comme un processus susceptible de déboucher possiblement sur la violence, car en pratique elle y conduit inéluctablement [Guibet Lafaye et Rapin, 2017, 144]. Le terme est difficile à utiliser à un niveau heuristique. En effet, la littérature tend à dissocier la radicalisation en tant que processus, du terrorisme ou de la violence en tant que produit [Guidère, 2016]. Pour ce dernier auteur, le terme de radicalisation reste singulier car son processus associe une composante à la fois intellectuelle et actionnelle. Aussi, 
la distinction entre sa définition théorique et sa perception phénoménologique dans la population est également à prendre en compte. On pose ici l'hypothèse que l'affinement théorique du terme n'est pas dénué de lien avec la transformation radicale des cultures matérielles associées à l'expression des pensées totalitaires. L'évolution sémantique de la radicalisation ne serait pas indépendante du développement des médias numériques (ou de ce que Mathieu Guidère nomme "l'internisation du terrorisme ») qui marque le basculement dans la violence par le canal d'internet.

Cette radicalisation virtuelle est une composante essentielle de son organisation techno-culturelle qui retentit plus fortement encore sur les grandes formes d'autorité sociale que les structures sectaires traditionnelles (les Témoins de Jéhovah, par exemple) qui sont dépourvues de la même maitrise technologique. Elle se distingue également des objectifs des nouveaux mouvements religieux et New Age ou des scientologues qui, tout en s'inscrivant dans des mécaniques sectaires, ont une meilleure maitrise des technologies de communication et de l'usage des réseaux sociaux. Dans l'organisation culturelle des diverses formes de radicalisation, la parole des institutions politiques, la parole scientifique et celle des médias sont directement touchées, défiées, et d'une intensité variable selon le type de pensées extrêmes (politique, religieuse, complotiste, alterscience). Ce qui implique pour elles d'opposer des contre-discours et de trouver des stratégies basées sur un activisme médiatique [Cardon et Granjon, 2013] afin de bloquer les fake news et les faits alternatifs découlant des énoncés totalitaires. La politique de communi- cation anti-radicalisation est malheureusement un échec [Schouten, 2016]. Mais l'article de Séraphin Alava, Divina Frau-Meigs et Ghayda Hassan montre la difficulté à qualifier les relations entre les médias sociaux et les processus de radicalisation menant à la violence. D'une part, le domaine de la radicalisation des « jeunes » en ligne est encore insuffisamment étudié, et d'autre part, le rôle précis des médias numériques dans le processus de radicalisation doit être exploré avec plus de soins. Il apparaît cependant que le jeu des algorithmes des réseaux sociaux, et la faiblesse des gate-keepers traditionnels sur Internet, tendent à favoriser des formes d'entresoi cognitif, et une plus faible exposition à des idées et propos qu'on ne partage pas [Bougnoux, 1995; Lawrence, Sides et Farrell 2010]³.

\section{Les radicalisations et la postmodernité}

La radicalisation opérant, souvent simultanément, dans plusieurs compartiments de la biographie individuelle, le pluriel rend mieux compte de ce processus qui peut concerner ensemble ou séparément, les domaines du religieux, du politique ou scientifique. En France, les « radicalisations », quelles que soient leur forme et leur origine, conduisent au détachement des valeurs républicaines ou démocratiques communément respectées $^{4}$. Ce détachement constitue une étape-clef dans les phénomènes d'embrigadement religieux de type djihadiste. On peut relier ce constat à un affaiblissement de la pensée moderne issue de l'humanisme des Lumières et à la théorie du postmatérialisme développée par Inglehart [1997]. Il explique le changement des valeurs d'une société à partir de son degré d'industrialisation, avec l'affirmation de valeurs individualistes associées 
au bien être social et à l'environnement au détriment de valeurs collectives plus traditionnelles. Ce retournement de la culture post-matérialiste [Galland et Roudet, 2005] n'est pas sans lien avec l'anxiété croissante provoquée par la dissolution des formes sociales et des spécificités nationales, analysée par Pierre-André Taguieff [2009]. Cela se traduit également par un nouveau rapport au savoir théorisé avec le postmodernisme, popularisé par une série d'auteurs comme Jean-François Lyotard [1979], Frederic Jameson au États-Unis et Stuart Hall au Royaume-Uni. Ils remettent en cause les deux grands récits de la modernité : l'émancipation du sujet rationnel d'un côté et l'histoire universelle du progrès de l'humanité de l'autre.

Nous avons isolé six marqueurs structurant la matrice du postmodernisme et posons l'hypothèse que cette empreinte est associée à certaines vulnérabilités cognitives.

Un premier marqueur identitaire s'appuie sur le différentialisme. Autrui définit une différence personnelle ou groupale à préserver, à valoriser, et suppose en conséquence des droits particuliers ; les affinités deviennent relatives et situées. Le risque d'une déconstruction du sujet politique se fait alors au profit de la valorisation des identités culturelles, religieuses ou sexuelles, comme le souligne Anne-Cécile Robert [2015].

Un second marqueur politique privilégie l'appartenance communautaire plutôt que nationale. On retrouve cela dans l'islamisme qui plaide pour une communauté soudée, supra et transnationale (l'oumma), tout en repoussant la forme stato- nationale et la démocratie moderne occidentale. En Syrie, la forme " impériale » adoptée par Daech permet à de jeunes Européens de s'engager pour un néocalifat qui précisément se joue des frontières tout en établissant ses bases territoriales. Parmi les 1200 à 1500 Français partis faire le djihad en Syrie et en Irak, un quart est constitué de convertis, d'autres, issus de familles des anciennes colonies et protectorats, peuvent être animés d'un « atavisme anti-français », et tous s'inscrivent dans une « oumma qui détient le rôle essentiel et qui s 'incarne par le califat dépositaire du sacré », écrit Farhad Khosrokhavar ${ }^{5}$.

Un troisième marqueur institutionnel est la logique qui promeut un vivre-ensemble basé sur l'expression individuelle plutôt que sur le principe de la représentation politique. L'article de Vincenzo Cicchelli et Sylvie Octobre consacré à la fictionnalisation des attentats chez les adolescents montre à quel point internet induit une conception de la démocratie davantage fondée sur la participation horizontale et sur une mise en doute continue de l'autorité officielle, que sur une parole institutionnelle ici largement dévaluée.

Un quatrième marqueur épistémologique, qui définit la réalité comme étant construite et multiple (hyperconstructivisme), plaide pour des relations de causes à effets souvent déterministes et cachées. Les théories du complot prolifèrent sur ce terreau, et retournent la modernité contre elle-même, en posant que le pouvoir des élus est un simulacre et qu'un « vrai » pouvoir, bien plus puissant, se tient nuisiblement derrière eux. Ce marqueur est également très prégnant dans l'enquête 
précitée, qui souligne le poids de l'image chez les jeunes et le manque de temps pour vérifier la véracité des informations reçues qui se succèdent de plus en plus vite. Une sorte de cercle vicieux se met alors en place où la multiplication des informations en circulation offre une potentialité accrue pour des lectures complotistes ou « alternatives » des événements.

Un cinquième marqueur de temporalité génère une action présente à partir de paris effectués sur l'avenir. Aussi, la perspective d'une hypersexualité tissée de polygamie et d'un paradis sexuel dans l'au-delà est un aspect de la rhétorique du recrutement djihadiste [Benraad 2018b].

Un sixième marqueur eschatologique est axé sur une fin de l'histoire apocalyptique. On peut citer en exemple la mystique du sacrifice des " shahids » (ceux qui se constituent en martyr du djihad) afin d'ouvrir les portes du paradis [Roy 2016].

Si on aborde culturellement la radicalisation comme l'expression de vulnérabilités cognitives liée à l'affaiblissement de la pensée moderne, le rapport aux nouvelles technologies et à l'Étatnation s'en trouve bouleversé. Il serait trop simple de concevoir le djihadisme comme une réponse traditionaliste et fondamentaliste au matérialisme des nouvelles technologies occidentales. La maîtrise des médias numériques des fondamentalistes est pointue et leurs préceptes religieux peuvent se revendiquer d'une préscience permettant à des prédicateurs d'affirmer la Révélation coranique de découvertes scientifiques contemporaines.

\section{L'État face au processus de radicalisation}

L'article de Julien Fragnon consacré aux outils gouvernementaux de lutte contre la radicalisation montre les limites et les difficultés des stratégies de communication publique de prévention du terrorisme et des messages axés sur la seule sécurité des populations. Les plans d'action visant à prévenir les départs en Syrie appuyés sur l'usage de l'émotion témoignent des limites curatives des outils développés par l'État. Cela nécessite plus largement la construction de contre-discours sur les réseaux sociaux et une politique d'intégration fondée sur l'acceptation des valeurs de la République. L'entretien croisé de Raphaël Josset et de Rudy Reichstadt montre la force et le dynamisme du militantisme complotiste utilisant les médias numériques.

L'Observatoire du conspirationnisme, la Fondation Jean Jaurès et l'Ifop ont récemment publié une enquête permettant d'estimer la pénétration des théories complotistes dans l'opinion publique française. Malgré des réserves méthodologiques qui appellent à la prudence ${ }^{6}$, celle-ci semble montrer une plus grande perméabilité des « jeunes », une corrélation avec les votes «populistes » et voit dans le conspirationnisme, un phénomène social majeur qui serait susceptible de toucher près d'un Français sur quatre. L'entretien croisé de Serge Galam et de Gérald Bronner souligne également les limites d'une résistance politique institutionnalisée. Une vision de lutte contre le radicalisme purement institutionnelle est ainsi vouée à l'échec, car les discours anti-conspirationnistes ou sur la radicalisation émanant d'universitaires et d'institutions n'empruntent pas les 
mêmes canaux et ne parviennent pas à atteindre leur cible [El Difraoui 2016].

Sans préjuger des aspects économiques et politiques, on peut supposer qu'avec une approche culturelle des processus de radicalisation, le traitement des vulnérabilités cognitives soit appréhendé en amont de la lutte contre les manifestations djihadistes, notamment leur forme terroriste, plutôt à partir de politiques culturelles et de politiques d'intégration adaptées à certains segments de la population, que de contre-discours dispersés et de faible rendement. Ceux-ci sont plus particulièrement inefficaces lorsqu'ils sont portés par des acteurs institutionnels ou médiatiques confrontés à un haut degré de défiance structurelle.

\section{$R \cdot E \cdot F \cdot E ́ R \cdot E \cdot N \cdot C \cdot E \cdot S$}

R. BADOUARD, Le désenchantement de l'Internet. Désinformation, rumeur et propagande, Limoges, FYP éditions, 2017.

D. BELL, The Cultural Contradictions of Capitalism, New York, Basic Books, 1976.

M. BENRAAD, Jihad. Des origines religieuses à l'idéologie, Paris, Éditions Le Cavalier Bleu, 2018a.

M. BENRAAD, «Les djihadistes sont-ils des frustrés sexuels ? », L'Opinion, 3 janvier 2018b. En ligne : https://www.lopinion.fr/edition/ international/myriam-benraad-djihadistes-sontils-frustres-sexuels-139818\#comments-anchor Accédé le 8 janvier 2018.

D. BOUGNOUX, La communication contre l'information, Paris, Hachette, 1995.

L. BOUVET, L'insécurité culturelle, Paris, Fayard, 2015.

G. BRONNER, La démocratie des crédules, Paris, PUF, 2013.

D. CARDON et F. GRANJON, Médiactivistes, Paris, Presses de SciencesPo, 2013.

P. CHASSIGNOLLES, « Contre la radicalisation », Archives de politique criminelle, vol. 1, $\mathrm{n}^{\circ} 38,2016$, p. 137-148.

X. CRETTIEZ, « Penser la radicalisation. Une sociologie processuelle des variables de l'engagement violent ", Revue Française de Science Politique, vol. 66/5, 2016, p. 709-727.

A. ELDIFRAOUI, Ledjihadisme, Paris, PUF, 2016. O. GALLAND et B. ROUDET (dir.), Les jeunes Européens et leurs valeurs: Europe occidentale, Europe centrale et orientale, Paris, La Découverte, 2005.

C. GUIBET-LAFAY et A.-J. RAPIN, « La 
radicalisation. Individualisation et dépolitisation d'une notion », Politiques de communication, vol. 1/8, 2017, p. 127-154.

M. GUIDÈRE, « Internet, haut lieu de la radicalisation », Pouvoirs, vol. 3/158, 2016, p. 115-123. R. INGLEHART, Modernization and postmodernization : Cultural, economic, and political change in 43 societies, Princeton, NJ, PUP, 1997. E. LAWRENCE, J. SIDES et H. FARRELL, « Self-Segregation or Deliberation? Blog Readership, Participation and Polarization in American Politics, Perspectives on Politics », vol.8/2, p. 141-157.

J.-F. LYOTARD, La Condition postmoderne. Rapport sur le savoir, Paris, Éditions de Minuit, 1979.

A.-C. ROBERT, «Assauts contre les Lumières », Le Monde Diplomatique, n731, février 2015. O. ROY, Le djihad et la mort, Paris, Le seuil, 2016. D. SCHOUTEN, U.S. Strategic Communications Against Islamic Fundamentalists, Thèse Monterey California : Naval Postgraduate School, 2016. https:/calhoun.nps.edu/bitstream/ handle/10945/48591/16Mar_Schouten_Dustin. pdf? sequence $=1$

K. STEINER et A. ÖNNEFORS (Eds.), Expressions of Radicalization - Global Politics, Processes and Practices, Palgrave, 2018.

P.-A. TAGUIEFF, La foire aux Illuminés. Ésotérisme, théorie du complot, extrémisme, Paris, Mille et une nuits, 2005.

P.-A. TAGUIEFF, « Une nouvelle illusion théorique dans les sciences sociales : la globalisation comme 'hybridation' ou 'métissage culturel' », Observatoire du communautarisme, 12/12/2009. I.-T. THOMSON, Culture Wars and Enduring America Dilemmas, University of Michigan Press, Michigan, 2010.

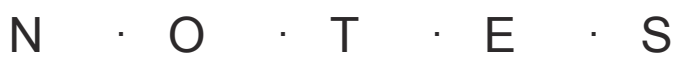

1. Les auteurs remercient Guy Saez pour sa relecture attentive et suggestions d'une précédente version. La mouture finale n'engage cependant que les auteurs.

2. Certaines dérives sectaires ont été très violentes (Charles Manson, Siège de Waco, Temple du Peuple au Guyana et Temple Solaire pour ne citer que quelques exemples).

3. Dans son livre sur Alain Soral, Bruno Di Mascio écrit : «La twitterisation de l'information tend ainsi à prendre une pensée radicale pour une pensée profonde parce qu'elle est facilement diffusable ». Cf. B. Di Mascio, Les souterrains de la démocratie. Soral, les complotistes et nous, Paris, Temps Présent, 2016, p. 111.

4. Ce paragraphe résume la proposition Vulnérabilités cognitives et retrait des valeurs républicaines déposée au CNRS par Virginie Tournay, Flora Chanvril, Marcel Kuntz, Guy Saez et Thierry Vedel lors du lancement de son appel pour analyser les attentats de janvier et de novembre 2015.

5. Farhad Khosrokhavar, «Les profils pluriels du djihadisme européen », Le Monde, 25 mars 2016.

6. «Une grande enquête sur le complotisme dans l'opinion publique française révèle une réalité alarmante », Observatoire du conspirationnisme, 07/01/2018 (http://www.conspiracywatch.info/unegrande-enquete-sur-le-complotisme-dans-lopinionpublique-francaise-revele-une-realite-alarmante.html). Si l'enquête est intéressante sur les liens établis entre sensibilité politique et croyances aux théories du complot, elle a le défaut de présupposer une familiarité avec des «théories du complot» bien connues des acteurs complotistes, comme des chercheurs, mais qui ne sont pas appréhendées de façon aussi cohérente par les profanes. On peut donc s'interroger sur la validité 
des réponses en termes d'adhésion à telle ou telle théorie fournie par des enquêtés qui... la découvrent au moment de l'enquête; et ce d'autant plus que l'offre de réponse élude le traditionnel « ne se prononce pas ». 